\title{
Co-relation of HIV, DM and Tobacco Habited in MDR-TB Suspected Patient
}

\author{
Dipali Gavali*, Binita Aring, Summiya Mullan and Akhlak Ahemad
}

Department Microbiology, Near TB Hospital, Vikas Gruh Road, Jamnagar, 361008, India

*Corresponding author

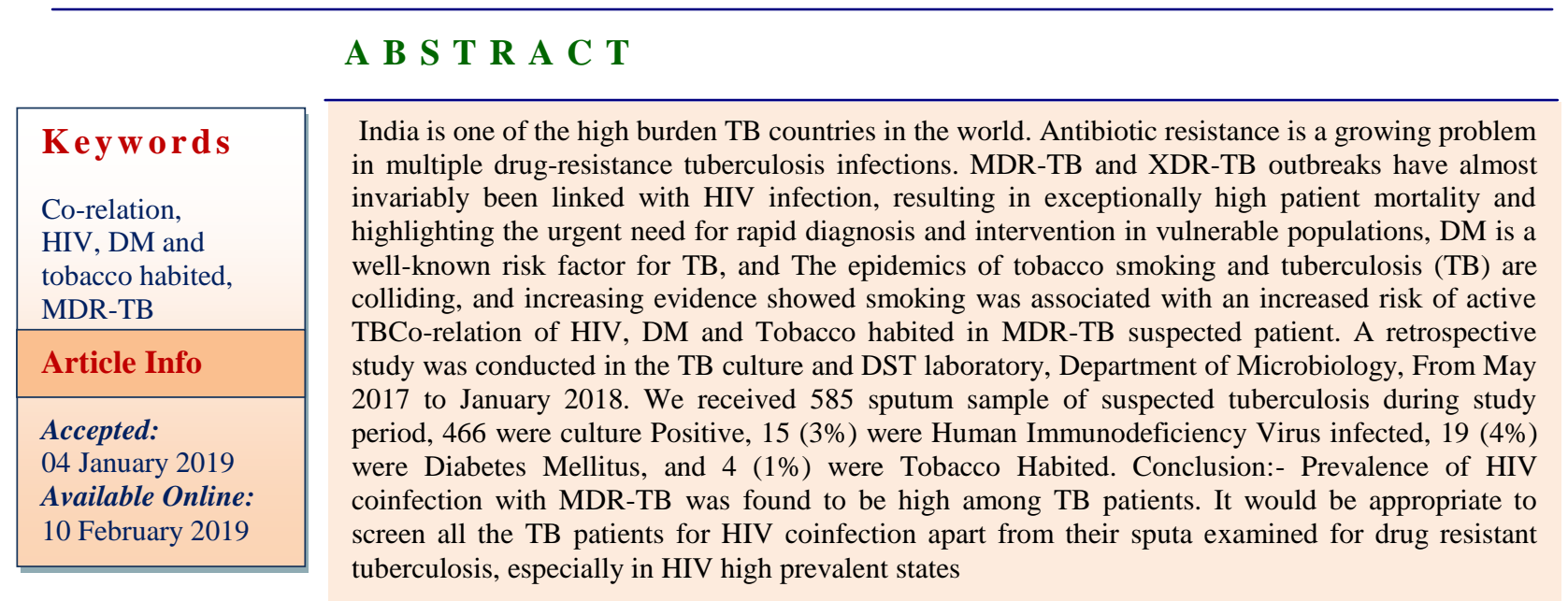

\section{Introduction}

Robert Koch isolated the tubercle bacillus in 1882 and proves its role in Tuberculosis. Tuberculosis is diseases caused by Mycobacterium Tuberculosis (MTB). Mycobacteria are non-motile, non sporing, non-capsulated, weakly gram positive, straight and slightly curved rod shapes bacteria which are obligate aerobes or microaerophilic. Mycobacterium transmitted by inhalation of droplets nuclei generated by cough and sneezing. The Diseases has a high prevalence in India, accounting for one fourth of the Tuberculosis (TB) cases in the world. India is one of the high burden TB countries in the world. ${ }^{[1]}$ According to the WHO Tuberculosis report 2014, there were 9 million incidence cases of TB globally. India having a share of $24 \%$ of the global burden. ${ }^{[1]}$ Tuberculosis is the second-most common cause of death from infectious diseases after those due to HIV/AIDS. ${ }^{[2]}$

Treatment of tuberculosis involves first and second-line anti-tuberculous drugs for drug susceptible and drug resistant cases respectively. During recent years there has been emergence of resistance to first-line TB drugs. Drug resistance in MTB develops by the selective growth of resistant mutants. Antibiotic resistance is a growing problem in 
multiple drug-resistance tuberculosis infections. Primary resistance occurs when a person becomes infected with a resistance strain of tuberculosis, but a person with fully susceptible tuberculosis may develop secondary (acquired) resistance during therapy because of inadequate treatment, not taking the prescribed regime appropriately (lack of compliance), or using low-quality medications. ${ }^{[3]}$

Multidrug Resistant Tuberculosis (MDR-TB) is defined when TB bacilli become resistant to both isoniazid and rifampicin or are monoresistant to rifampicin. Pre-Extensively DrugResistant Tuberculosis (Pre XDR-TB) is defined as resistance to rifampicin and/or isoniazid with additional resistance to secondline drugs i.e. to any Fluoroquinolone (FQ), or to at least one of the three injectable second-line drugs [Amikacin, Kanamycin, and Capreomycin (AM)]. Thus Pre XDR-TB consists of two subgroups i.e. 1) MDR-TB with FQ resistance [Pre XDR-TB (FQ)], 2) MDR-TB with AM resistance [Pre XDR-TB (AM)]. Extensively Drug-Resistant Tuberculosis (XDR-TB) is defined as resistance to rifampicin and/or isoniazid with additional resistance to second-line drugs i.e. to any $\mathrm{FQ}$, and to at least one of the three injectable second-line drugs (AM).

World Health Organisation had estimated about 480000 MDR-TB cases in 2014 out of which only 123000 were detected. About 110000 patients of MDR-TB were put on treatment ${ }^{[4]}$. Of these around 24000 cases of MDR-TB were diagnosed and initiated on therapy in India. ${ }^{[1]}$ World Health Organization (WHO) recommends baseline testing for FQ and AM only among the second-line drugs due to availability of standardised tests and as these two groups of drugs are an important part of MDR-TB treatment regimens across the globe. Subsequent transmission of resistant bacilli is facilitated by inadequate infection control, especially in congregate settings. MDR-TB and XDR-TB outbreaks have almost invariably been linked with HIV infection ${ }^{[5,6]}$, resulting in exceptionally high patient mortality and highlighting the urgent need for rapid diagnosis and intervention in vulnerable populations.

DM is a well-known risk factor for TB. It increases the risk of developing active TB by a factor of 2-3 compared with the normal population $^{[7]}$. There are also reports of DM patients with TB being more likely to develop drug-resistant $\mathrm{TB}^{[10,11]}$, although the numbers of patients reported in these studies are small, and there is little information about whether $\mathrm{DM}$, duration of disease and control of DM have any association with drug-resistant TB.

The epidemics of tobacco smoking and tuberculosis (TB) are colliding, and increasing evidence showed smoking was associated with an increased risk of active TB. An understanding of the epidemiological relationship between smoking and tuberculosis is important because both smoking and tuberculosis cause extensive morbidity and mortality worldwide. Compared with those who have never smoked, it is estimated that people who smoke have approximately twice the risk of both Mycobacterium tuberculosis infection ${ }^{[12]}$ and active tuberculosis. ${ }^{[13]}$

Becton, Dickinson and Company (BD) developed a new system called Mycobacteria Growth Indicator Tube (MGITTM), which is non-radiometric and offers the rapid, sensitive and reliable methods of testing as the BACTEC 460 TB System. BBL MGITTM System is the manual system while BACTEC MGIT 960 (MGIT 960) is the fully automatic system for detection of mycobacterial growth and drug susceptibility testing of $\mathrm{M}$. tuberculosis. ${ }^{[8,9]}$ 
In our study, we aimed to find the prevalence of Pre XDR-TB and XDR-TB amongst newly diagnosed cases of pulmonary MDR-TB who had never been previously treated with second-line drugs and with coinfection/comorbidities like HIV-TB, DM-TB and tobacco habited-TB by using of BACTEC MGIT 960 (MGIT 960) instrument.

\section{Aims and Objectives}

We studied the Co-relation of HIV, DM and Tobacco habited in MDR-TB suspected patient.

\section{Patients were grouped in to}

(1) MDR-TB with HIV, DM and Tobacco Habited

(2) Pre XDR -TB with HIV, DM and Tobacco Habited

(3) XDR-TB HIV, DM and Tobacco Habited

The final data was reported as prevalence of MDR-TB, Pre XDR-TB and XDR-TB in HIV, DM and Tobacco habited patient cases of pulmonary MDR-TB patient.

\section{Materials and Methods}

A retrospective study was conducted in the TB culture and DST laboratory, Department of Microbiology, in our Hospital, From May 2017 to January 2018. According to History of Human Immunodeficiency virus (HIV), Diabetes (DM) and Tobacco habited we had divided the patient. There were 2 sputum samples, one spot supervised and one early morning collected in sterile screw cap wide mouth falcon tube and transported from various centers to TB culture-DST laboratory by courier with cold chain maintained. All sputum samples were processed following the standard NALC-NaOH method for digestion, decontamination, and concentration ${ }^{[14,15]}$. The concentrated sediment was resuspended in about 2 to $3 \mathrm{ml}$ phosphate buffer ( $\mathrm{pH} \mathrm{6.8)} \mathrm{and}$ mixed thoroughly. Use the resuspended pellet for making smears and for inoculation of MGIT tubes and other media, than according to result follow the direct microscopy, culture and subculture. The use of BACTEC MGIT 960 drug susceptibility testing was done and according to the sensitivity of drug they divided in to MDR-TB, Pre -XDR TB and XDR-TB. We performs second line drug susceptibility testing (Moxifloxacin, Levofloxacin, Amikacin and kanamycin)

\section{Principle of the BACTEC ${ }^{\text {TM }}$ MGIT ${ }^{\text {TM }} 960$ System}

The MGIT (Mycobacteria Growth Indicator Tube) consists of liquid broth medium that is known to yield better recovery and faster growth of mycobacteria. MGIT tube contains an oxygen-quenched fluorochrome, tris 4, 7diphenyl-1, 10-phenonthroline ruthenium chloride pentahydrate, embedded in silicone at the bottom of the tube. During bacterial growth within the tube, the free oxygen is utilized and is replaced with carbon dioxide. With depletion of free oxygen, the fluorochrome is no longer inhibited, resulting in fluorescence within the MGIT tube when visualized under UV light. The intensity of fluorescence is directly proportional to the extent of oxygen depletion.

If the test drug is active against the isolated mycobacteria, it will inhibit the growth and thus there will be suppression of fluorescence, while the growth control will grow uninhibited and will have increasing fluorescence.

Growth is monitored by the BACTEC 960 instrument which automatically interprets results as susceptible or resistant. Record "Susceptible (S)", "Resistant (R) ", or "Test Failed (TF)" on the internal lab worksheet/book and the lab requisition form 
(if applicable). Also, keep the MGIT printouts for all DST results with the patient records.

\section{Data collection}

We received the sample from the various district for the drug susceptibility testing so, they send the history of the patient like Treatment history, HIV, DM, Tobacco Habits, Alcohol abuse, and gene xpert result for rifampicin resistance.

According to this we study on correlation of HIV-TB, DM-TB and Tobacco-TB in various group of TB Like MDR-TB, Pre XDR-TB and XDR-TB.

\section{Results and Discussion}

We received 585 sputum sample of suspected tuberculosis during study period, 466 were culture Positive. Out of 466 culture positive sputum samples of pulmonary tuberculosis, 15 (3\%) were Human Immunodeficiency Virus (HIV) infected of which 11 had MDRTB, 2 had Pre XDR-TB and 2 had XDR-TB (Table 1).

19 (4\%) were Diabetes Mellitus (DM) of which 12 had MDR-TB, 6 had Pre XDR-TB and 1 had XDR-TB (Table 2).

4 (1\%) were Tobacco Habited of which 1 had MDR-TB, 2 had Pre XDR-TB and 1 had XDR-TB.

Table.1 Shows HIV Infected MDR-TB patients

\begin{tabular}{|l|c|c|c|}
\hline \multicolumn{1}{|c|}{ Category } & $\begin{array}{c}\text { MDR } \\
\text { [No. of Patient] }\end{array}$ & $\begin{array}{c}\text { Pre XDR TB } \\
\text { [No. of Patient] }\end{array}$ & $\begin{array}{c}\text { XDR } \\
\text { [No. of Patient] }\end{array}$ \\
\hline Male & 9 & 2 & 1 \\
\hline Female & 2 & 0 & 1 \\
\hline
\end{tabular}

In table 1 we can see from above 15 were HIV Infected MDR TB patients of which 11 had MDR-TB, 2 had Pre XDR-TB [FQ], 2 had XDR-TB.

Graph.1 HIV Infected MDR TB patients

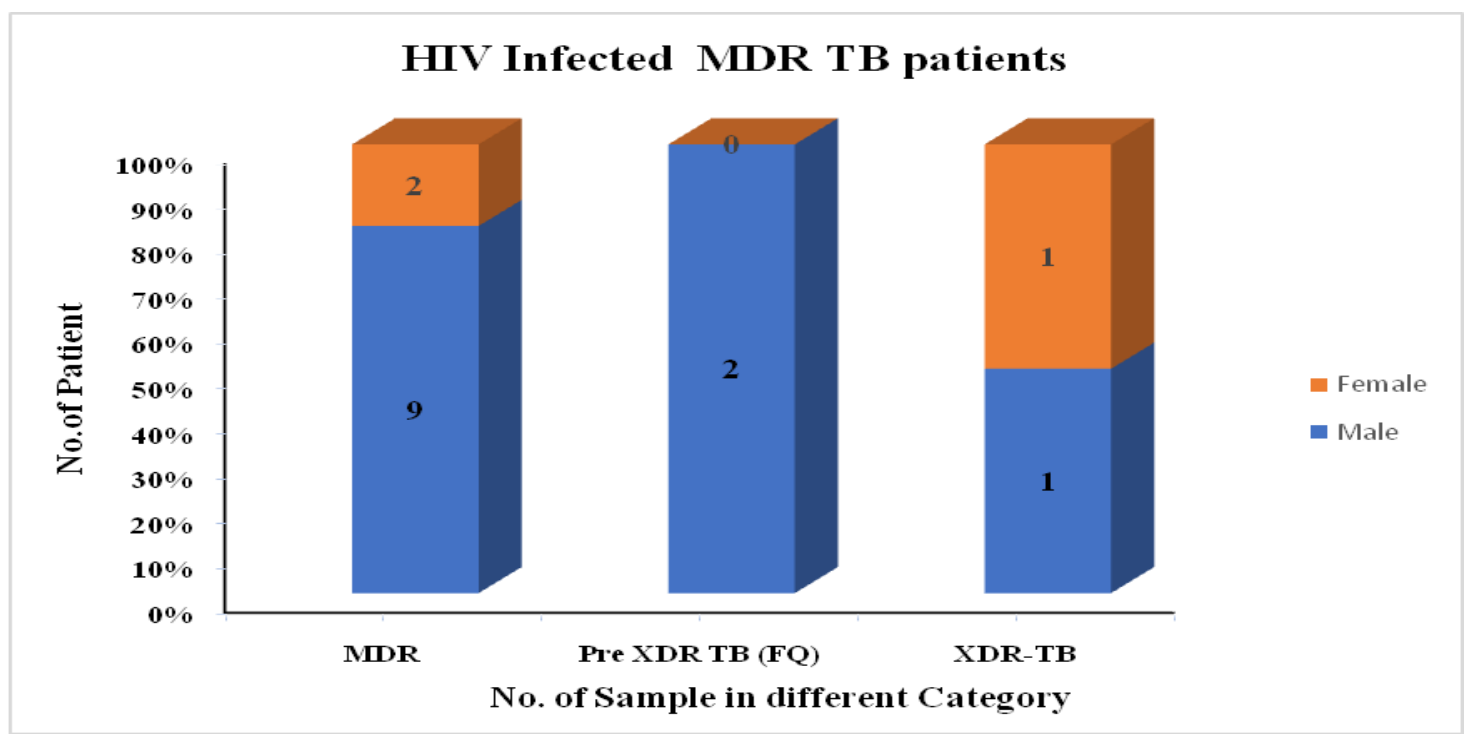


Table.2 Shows diabetes MDR-TB patients

\begin{tabular}{|l|c|c|c|}
\hline \multicolumn{1}{|c|}{ Category } & $\begin{array}{c}\text { MDR } \\
\text { [No. of Patient] }\end{array}$ & $\begin{array}{c}\text { Pre XDR TB } \\
\text { [No. of Patient] }\end{array}$ & $\begin{array}{c}\text { XDR } \\
\text { [No. of Patient] }\end{array}$ \\
\hline Male & 11 & 5 & 0 \\
\hline Female & 1 & 1 & 1 \\
\hline
\end{tabular}

In table 2 we can see from above 19 were Diabetes, 12 had MDR-TB, 6 had Pre XDR-TB (FQ), 1 had XDR- TB.

Graph.2 Diabetic MDR TB patients

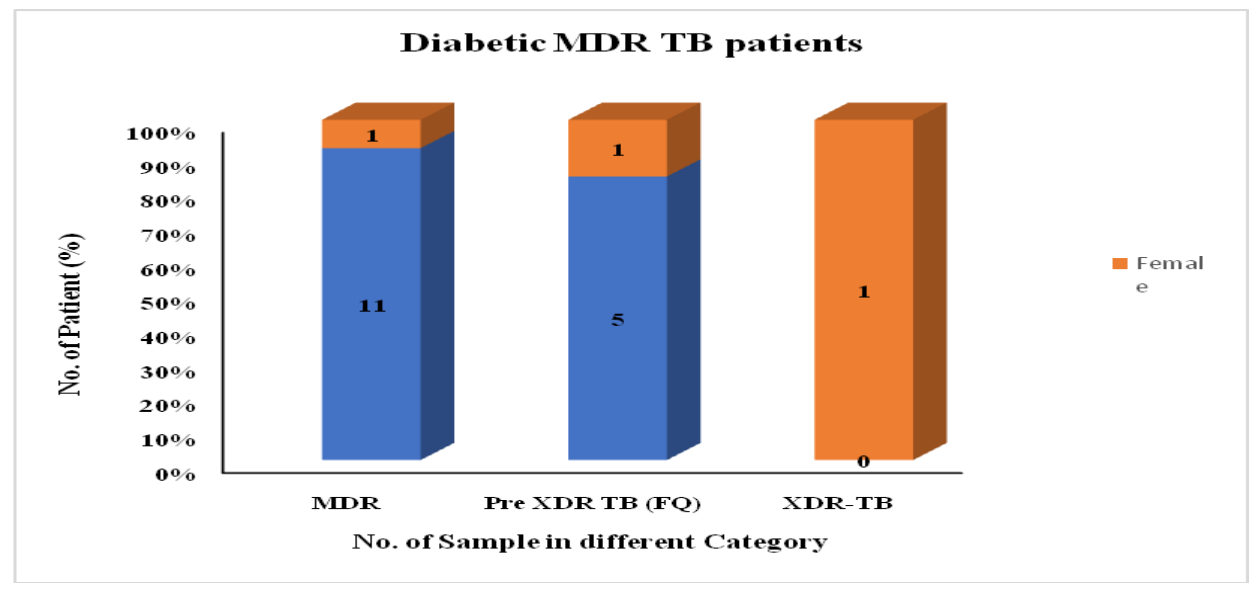

Table.3 Shows Tobacco abused MDR-TB patients

\begin{tabular}{|l|c|c|c|}
\hline \multicolumn{1}{|c|}{ Category } & $\begin{array}{c}\text { MDR } \\
\text { [No. of Patient] }\end{array}$ & $\begin{array}{c}\text { Pre XDR TB } \\
\text { [No. of Patient] }\end{array}$ & $\begin{array}{c}\text { XDR } \\
\text { [No. of Patient] }\end{array}$ \\
\hline Male & 1 & 2 & 1 \\
\hline Female & 0 & 0 & 0 \\
\hline
\end{tabular}

In table 3 we can see from above 4 were Tobacco Habited, 1 were MDR-TB, 2 were Pre XDR-TB (FQ), 1 were XDR- TB.

Graph.3 Tobacco habited MDR TB patients

Tobacco Habited MDR TB patients

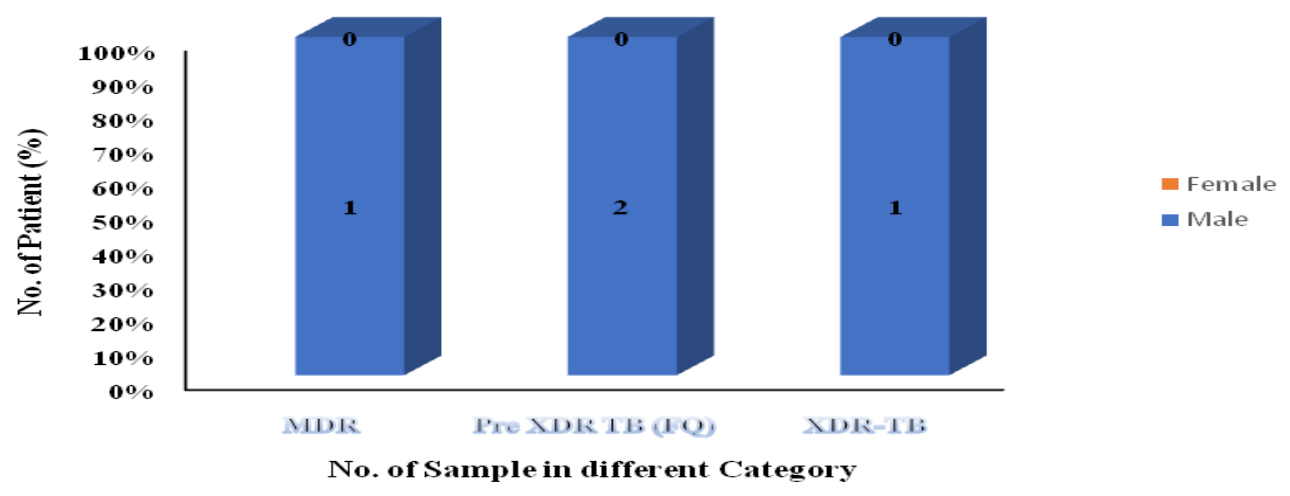


Table.4 Comparison of HIV-TB coinfection among MDR-TB

\begin{tabular}{|c|l|}
\hline HIV-TB Coinfection [\%] & \multicolumn{1}{|c|}{ Study } \\
\hline 27.9 & Balaji (2010) $^{[17]}$ \\
\hline 13.9 & Rajasekharan $(\mathbf{2 0 0 9})^{[18]}$ \\
\hline 4.42 & Deivanayagam $(\mathbf{2 0 0 2})^{[21]}$ \\
\hline 4 & Sameer Adwani $(\mathbf{2 0 1 6})^{[16]}$ \\
\hline 3 & Present Study $(\mathbf{2 0 1 7})$ \\
\hline
\end{tabular}

$>$ We studied common comorbidities of HIV among MDR-TB patients. 15 of our study patients were HIV infected which is approximately $3 \%$.

$>$ This is higher than the reported $2009 \mathrm{NACO}^{[24]}$ adult HIV prevalence of $0.31 \%$, though HIV-TB co-infection data reported from India is variable.

> A study of Balaji et al., Tamil nadu $2010{ }^{[17]}$ were tested for HIV most patients with HIV were not on antiretroviral treatment, and Rajasekharan et al., thoracic medicine, Chennai $2009^{[18]}$ conduct study on Prevalence of HIV coinfection with MDR-TB was found to be high among chronic TB patients had reported higher prevalence of $27.9 \%$ and $13.9 \%$ HIV MDR-TB co-infected patients.

$>$ In a study conducted in Deivanayagam et al., govt. hospital thoracic medicine, Chennai 2002 ${ }^{[21]}$ and Sameer Adwani Mumbai et al., 2016, were HIV seropositivity among MDR-TB was reported as $4.42 \%$ and $4 \%{ }^{[16]}$ similar with our results.

Table.5 Comparison of correlation of TB-Diabetes among MDR-TB

\begin{tabular}{|c|l|}
\hline$T B-D M$ Correlation $[\%]$ & \multicolumn{1}{c|}{ Study ${ }^{[19]}$} \\
\hline 31.6 & Fisher- Hoch $\operatorname{SP}(2008)^{[19]}$ \\
\hline 16.6 & Fengling Mi (2014) ${ }^{[20]}$ \\
\hline 11 & Sameer Adwani(2016) \\
\hline 4 & Present Study (2017) \\
\hline
\end{tabular}

In present study Prevalence of TB-diabetes were 19 (4\%), this were more lower than World Health Organization, Geneva shows in India Diabetes accounts for $14.8 \%$ of pulmonary tuberculosis. ${ }^{25]}$

$>$ Lower prevalence in our study is due to observed prevalence does not reflect the prevalence in the community since this was a tertiary care centre and in general, referral basis can lead to wide variation in the observed prevalence amongst different centres.

> Present study compared with International studies by SP Fisher-Hoch et al., Brownsville USA (2008)on association between tuberculosis (TB) and diabetes is re-emerging with the epidemic of type 2 diabetes (T2DM) reported higher prevalence DM of 31.6\% in MDR-TB ${ }^{[19]}$. Fengling Mi et al., Beijing TB and thoracic tumour research institute, China (2014) study on is resistance to anti-tuberculosis drugs associated with type 2 diabetes mellitus reported the prevalence of DM as $16.6 \%{ }^{[20]}$.Sameer Adwani et al., TNMC and BYL Nair Hospital, Mumbai (2016) ${ }^{[16]}$ reported prevalence of DM of $11 \%$ in MDR-TB patients.

$>$ Optimal treatment of these conditions is essential with appropriate MDR therapy.

Table.6 Comparison of correlation of TB-Tobacco among MDR-TB

\begin{tabular}{|l|l|}
\hline Tobacco-TB Correlation [\%] & Study \\
\hline $\mathbf{2 8 . 8}$ & Zhang et al., 2017 \\
\hline $\mathbf{4 . 6}$ & Gegia et al., 2014 22$]$ \\
\hline $\mathbf{1}$ & Present Study (2017) \\
\hline
\end{tabular}

$>$ In present study prevalence of TB-Tobacco were 4 (1\%), this were very lower than World Health Organization, Geneva shows in India $40 \%$ of TB burden may be attributed to smoking. ${ }^{[26]}$ At present study we focused on only Pre XDR TB and XDR -TB and also study on DST laboratory sample referred by other districts so, not availability of proper information our study prevalence is lower than other study.

$>$ H. Zhang et al., study on a dose- response relationship of smoking with tuberculosis infection, $2017^{[23]}$ and M. Gegia et al., study on Tobacco smoking and tuberculosis treatment outcomes, 2014 ${ }^{[22]}$ both study done on tobacco - tuberculosis correlation so, their prevalence were higher than our study. 
Present study was done to know the baseline pattern of drug resistance among the pulmonary MDR-TB patients who had not been previously exposed to second line drugs in form of anti-tuberculous therapy. As per WHO recommendations, baseline second-line DST to FQ and AM was studied only.

Total 585 sputum samples, 466 had tuberculosis infection, 293 were Multidrug resistance Tuberculosis, 151 were Pre Extensively drug resistance Tuberculosis and 22 were Extensively drug resistance Tuberculosis (Table 1-6).

In conclusion, we studied the prevalence of Pre XDR-TB and XDR-TB among MDR-TB patients, which were $32.4 \%$ and $4.7 \%$ respectively. The high prevalence of Pre XDR-TB (FQ) is alarming and of concern in management of MDR-TB. Indiscriminate use of fluoroquinolone should be stopped. These patients should be evaluated for the presence of comorbities like HIV and DM and treated appropriately.

Prevalence of HIV coinfection with MDR-TB was found to be high among TB patients. It would be appropriate to screen all the TB patients for HIV coinfection apart from their sputa examined for drug resistant tuberculosis, especially in HIV high prevalent states.

For Diabetes -TB Correlation prospective research is needed on the association between $\mathrm{DM}$ and drug-resistant $\mathrm{TB}$, and this research will need to factor in other determinants of drug resistance in order to better understand the interactions between the two diseases.

Tobacco smoking increases the risk of a poor treatment outcome. Our findings support that cigarette smoking was independently associated with increased risk of TB infection. More attention should be attached to smokers, especially elderly smokers. In addition, active case finding among populations with specific risks such as smokers, close contacts, diabetes should be strengthened as well.

\section{References}

Balaji V, Daley P, Anand AA, Sudarsanam T, Michael JS, Sahni RD, et al., Risk factors for MDR and XDR-TB in a tertiary referral hospital in India. PLoS One 2010; 5: e9527.

Deivanayagam $\mathrm{CN}$, Rajasekaran S, Venkatesan R, Mahilmaran A, Ahmed PR, Annadurai S, et al., Prevalence of acquired MDR-TB and HIV coinfection. Indian J Chest Dis Allied Sci 2002; 44: 23742.

den Boon S, van Lill SW, Borgdorff MW, Verver S, Bateman ED, Lombard CJ, et al., Association between smoking and tuberculosis infection: a population survey in a high tuberculosis incidence area. Thorax. 2005 Jul; 60(7):555-7. doi: http://dx.doi.org/10.1136/thx.200.4030 924 PMID: 15994262

Department of AIDS control. Ministry of Health and Family Welfare. Annual Report 2009-10.Available from http://www.naco.gov.in/upload/REPO RTS/ NACO_AR_English\%202009. 10.pdf accessed on 9th March 2016.

Dolin, (edited by) Gerald L. Mandell, john E. Bennett, Raphael (2010). Mandellduglas, and benntt's principles and practice of infectious diseases $\left(7^{\text {th }}\right.$ ed.) Philadelphia, PA: Churchill Livingstone/Elsevier. Pp. Chapter 250.

Fisher-Hoch SP, Whitney E, McCormick JB, Crespo G, Smith B, Rahbar MH, et al., Type 2 diabetes and multidrugresistant tuberculosis. Scand J Infect Dis. 2008; 40: 888-93.

Fisher-Hoch SP, Whitney E, McCormick JB, Crespo G, Smith B, Rahbar MH, et 
al., Type 2 diabetes and multidrugresistant tuberculosis. Scand J Infect Dis 2008; 40(11-12): 888-93.

Gandhi NR et al., Extensively drug-resistant tuberculosis as a cause of death in patients co-infected with tuberculosis and HIV in a rural area of South Africa. Lancet, 2006, 368:1575-1580.

Jeon CY, Murray MB. Diabetes mellitus increases the risk of active tuberculosis: a systematic review of 13 observational studies. PLoS Med. 2008; 5:e152.

Kent PT, Kubica GP. 1985. Public health microbiology, a guide for the level III laboratory. Centers for Disease Control, Division of Laboratory Training and Consultation, Atlanta, GA.

Leung CC, Li T, Lam TH Yew WW, Law WS, Tam CM, et al., Smoking and tuberculosis among the elderly in Hong Kong. Am J Respir Crit Care Med. 2004 Nov 1; 170(9):1027-33. doi: http://dx.doi.org/10.1164/rccm. 200404512OC PMID: 152822

Medea Gegia, Mattew J Magee, Rusell R Kempker, Iagor Kalandadze, Tsira Chakhaia, Jonathan E Golub and Henry M Blumberg. Tobacco smoking and tuberculosis /BLT. treatment outcomes: a prospective cohort study in Georgia.2014.http://dx.doi.org/ 10.2471/BLT.14.147439.

Mi F, Jiang G, Du J, Liang Li, Yue W, Harries AD et al., Is resistance to antituberculosis drugs associated with type 2 diabetes mellitus? A register review in Beijing, China. Global Health Action 2014; 7:10.3402/ gha.v7.24022. 22

O'Brian R (1994). “Drug-resistant tuberculosis: etiology, management and prevention". Semin Respir Infect 9 (2):104-12. PMID 7973169.

Rajasekaran S, Chandrasekar C, Mahilmaran
A, Kanakaraj K, Karthikeyan DS, Suriakumar J. HIV coinfection among multidrug resistant and extensively drug resistant tuberculosis patients - a trend. J Indian Med Assoc 2009; 107(05): 281-2, 284-6.

Roberts GD, Goodman NL, Heifets L, et al., Evaluation of the BACTEC radiometric method for recovery of mycobacteria and drug susceptibility testing of Mycobacterium tuberculosis from acid-fast smear- positive specimens. J Clin Microbiol. 1983; 18: 689-696.

Sameer Adwani et al., JKIMSU, Prevalence of Pre-Extensively Drug-Resistant Tuberculosis (Pre XDR-TB) and Extensively Drug-Resistant Tuberculosis (XDR-TB) among Pulmonary Multidrug Resistant Tuberculosis (MDR-TB) at a Tertiary care center in Mumbai, Vol. 5, No. 3, July-September 2016.

Siddiqi SH, Libonati JP, Middlebrook G. Evaluation of a rapid radiometric method for drug susceptibility testing of Mycobacterium tuberculosis. J Clin Microbiol. 1981; 13: 908-912.

Siddiqi SH, Rüsch-Gerdes S. 2006. MGIT procedure manual. Foundation for Innovative New Diagnostics (FIND), Geneva, Switzerland.

Tatar D, Senol G, Alptekin S, Karakurum C, Aydin M, Coskunol I, et al., Tuberculosis in diabetics: features in an endemic area. Jpn J Infect Dis. 2009; 62: 423-7.

Wells CG et al., HIV infection and multidrugresistant TB - the perfect storm. Journal of Infectious Diseases, 2007, 196(Suppl.1): S86-107.

World Health Organisation, Global tuberculosis report 2015. Available from http://apps.who.int/iris/ bitstream/10665/191102/1/ 97892415 
65059_eng.pdf?ua $=1$ accessed on 9th March 2016

World Health Organisation, MultidrugResistant Tuberculosis (MDR-TB), 2015 Update. Available from http://www.who.int/tb/challenges/mdr/ mdr_tb_factsh eet.pdf accessed on 9th March 2016.

World Health Organization (2016)." The sixteenth global report on tuberculosis" (PDF), Geneva shows in
India Diabetes report 2016. www.who.int/tb and www.who.int/tobacco. Zhang H, Xin H, Li X, Li H, Li M, Lu W, et al., (2017) A dose-response relationship of smoking with tuberculosis infection: A crosssectional study among 21008 rural residents in china. PLoS ONE. 12(4): e0175183. Doi:10.1371/journal.pone. 0175183.

\section{How to cite this article:}

Dipali Gavali, Binita Aring, Summiya Mullan and Akhlak Ahemad. 2019. Co-relation of HIV, DM and Tobacco Habited in MDR-TB Suspected Patient. Int.J.Curr.Microbiol.App.Sci. 8(02): 278-286. doi: https://doi.org/10.20546/ijcmas.2019.802.033 